\title{
Niveles de procesamiento: Un marco para la investigación sobre la memoria (*)
}

\section{Fergus I. M. Craik y Robert S. Lockhart}

Universidad de Toronto

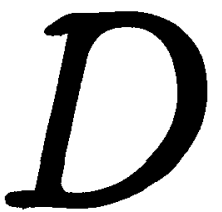

Durante la última década los modelos de la memoria humana han estado dominados por el concepto de la existencia de «almacenes» y de la transferencia de información entre ellos. Un criterio fundamental para distinguir a unos almacenes de otros ha sido el de sus diferentes características de retención. De esta forma, las propiedades temporales de la información almacenada han jugado un doble papel: además de constituir el fenómeno básico que había que explicar se utilizaban para generar los constructos teóricos en términos de los cuales se formulaba la explicación. Esta aparente circularidad se evitaba mediante la especificación de otras propiedades adicionales de los almacenes (como su capacidad y características de codificación), con lo que se les caracterizaba con independencia de los fenómenos que había que explicar. Los constructos así formulados se han utilizado para dar cuenta de los datos recogidos en diversos paradigmas y condiciones experimentales. El concepto esencial en que se basan tales explicaciones es la noción de que la información se transfiere de un almacén a otro, y los modelos de transferencia de un almacén a otro deben diferenciarse, por lo me. nos relativamente, de otras explicaciones que asocian diversas características de retención con los cambios cualitativos que tienen lugar en el código de la memoria.

En este artículo vamos a hacer tres cosas: a) examinar las razones que hay para proponer modelos en términos de múltiples almacenes; b) cuestionar si son adecuados o no; c) proponer un marco alternativo en términos de niveles de procesamiento. Mantenemos que el trazo de la memoria puede entenderse como un subproducto del análisis perceptivo y que su persistencia constituye una función positiva de la profundidad con la que se analiza el estímulo. Los estímulos también pueden retenerse durante intervalos de tiempo breves, a través de un procesamiento continuado a una profundidad constante. Estos puntos de vista ofrecen un nuevo camino para interpretar los datos existentes y proporcionan un marco heurístico para posteriores investigaciones.

\section{Los modelos multialmacén Alegatos a su favor}

Cuando se considera al hombre como un procesador de la información (Miller.

(*) Con autorización del autor y editor. Tomado de Journal of Verbal Learning and Verbal Behavior. 11, $671-684$ (1972)

Copgright (1972) by Academic Press. Reprinted by permission. 
1956; Broadbent, 1958) parece necesario proponer la existencia de «mecanismos de mantenimiento» 0 "almacenes de memoria» en varios puntos del sistema. Por ejemplo, Broadbent (1958), basándose en sus estudios sobre escucha dicótica, propuso que la información debe mantenerse de forma transitoria antes de entrar en un canal de procesamiento de capacidad limitada. Los items podrían mantenerse en esa memoria a corto plazo repasándolos, después de haberlos percibido, en ese mismo sistema transitorio de almacenamiento. De ahí la información se transferiría a un almacén a largo plazo, de carácter más permanente, en el que podría retenerse. Waugh y Norman (1965), Peterson (1966), Atkinson y Shiffrin (1968) han desarrollado y ampliado las ideas de Broadbent. De acuerdo con el modelo más extendido (Murdock, 1967) se acepta ahora ampliamente que la memoria puede clasificarse en tres niveles de almacenamiento: los almacenes sensoriales, la memoria a corto plazo (MCP) y la memoria a largo plazo (MLP). Puesto que ha habido cierta ambigüedad en el modo de utilizar los términos en este área, seguiremos la convención de utilizar las siglas MCP y MLP para referirnos a situaciones experimentales, y los términos «almacén a corto plazo» (ACP) y "almacén a largo plazo" (ALP) para designar los dos sistemas de almacenamiento a los que nos estamos refiriendo.

Los estímulos pueden entrar en los almacenes sensoriales prescindiendo de que el sujeto los preste o no atención; es decir, los almacenes sensoriales son "preatentivos" (Neisser, 1967). El imput se representa de una forma bastante literal y puede ser tachado por otros imputs que provengan de la misma modalidad (Neisser, 1967; Crowder y Morton, 1969); otros rasgos que distinguen a los registradores sensoriales de los almacenes posteriores son su naturaleza específica en cuanto a la modalidad sensorial, la capacidad relativamente grande de esos almacenes y la breve duración de sus contenidos.
El hecho de atender al material del registrador sensorial equivale a sacarlo de él y transferitlo al ACP. En este, los items verbales se codifican de forma fonémica (Schulman, 1971) o en términos lingüísticos audioverbales (Atkinson y Shiffrin, 1968). Además el ACP se distingue de las memorias sensoriales por el carácter limitado de su capacidad (Miller, 1956; Broadbent, 1958) y por el hecho de que la información se pierde principalmente a través de un proceso de desplazamiento (Waugh y Norman, 1965), así como por el hecho de que la velocidad de olvido del ACP es más lenta: 5-20 segundos frente a 1/4-2 segundos, que son las estimaciones para el almacén sensorial. Aunque la mayor parte de las investigaciones se han centrado en el ACP verbal, hay pruebas de que también puede mantenerse una información «representacional», más literal durante un corto plazo (Posner, 1967), aunque la relación entre esos almacenes de modalidad específica y el ACP verbal aún no está clara.

Las diferencias entre el ACP y el ALP están bien estudiadas. Mientras que el ACP tiene una capacidad limitada, el ALP no tiene límite conocido; los items verbales suelen codificarse con arreglo a un formato fonético en el ACP, mientras que en el ALP se codifican sobre todo en términos de sus rasgos semánticos (Baddeley, 1966); el olvido del ACP se completa en 20 segundos o menos, mientras que el olvido del ALP es muy lento o el material no se olvida en absoluto (Shifrrin y Atkinson, 1969). En el paradigma de recuerdo libre, suele pensarse que los últimos items se recuperan del ACP y los primeros del ALP; ahora sabemos que hay diversas variables que afectan a uno de estos componentes de recuperación sin afectar al otro (Glanzer, 1972). Los estudios clínicos (Milner, 1970; Warrington, 1971) proporcionan también pruebas persuasivas de la dicotomía ACP/ALP. Los rasgos que distinguen a los tres niveles de almacenamiento se resumen en el cuadro 1 . 


\section{CUADRO 1}

\section{DIFERENCIAS COMUNMENTE ACEPTADAS ENTRE LOS TRES ESTADIOS DE MEMORIA VERBAL}

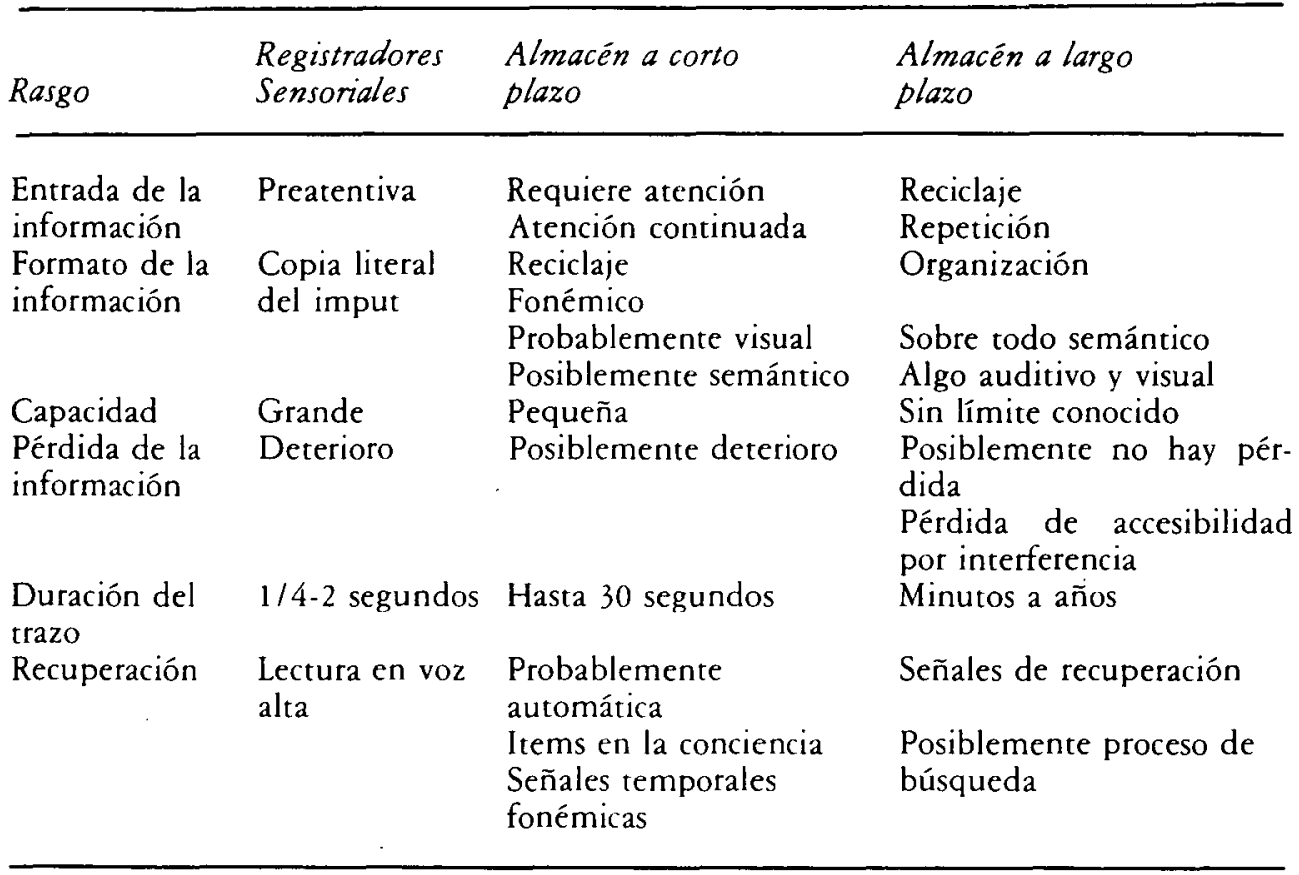

No es difícil de entender la atracción del enfoque de las «cajas». Estos modelos multialmacén son aparentemente específicos y concretos; la información fluye por vías bien reguladas entre los almacenes cuyas características resultan atractivamente intuitivas; pueden provocarse mediante un experimento sus propiedades y describirse conductual o matemáticamente. Parece que lo único que queda es especificar las propiedades de cada componente de una forma más precisa y elaborar las funciones de transferencia con más exactitud.

A pesar de estos puntos a su favor, los almacenes resultan menos tangibles cuando se examinan con más detalle las pruebas a favor de los modelos multialmacén. El papel cada vez más importante de los «procesos de control» en las formulaciones más recientes (Atkinson y Shiffrin, 1971) constituye una señal de alarma. En la pró- xima sección vamos a reconsiderar de una forma más crítica la validez de las nociones de estos modelos.

\section{Alegatos en contra}

Ha habido críticos que han formulado objeciones generales contra el enfoque multialmacén (Melton, 1963; Murdock, 1972). Otros han criticado ciertos aspectos de la formulación. Por ejemplo, Tulving y Patterson (1968) criticaron la noción de que la información se transfiere de un almacén a otro. De forma similar, Shallice y Warrington (1970) presentaron pruebas contrarias a la idea de que la información debe «pasar» necesariamente a través del ACP para entrar en el ALP.

Según nuestro punto de vista, los criterios recogidos en la sección anterior no 
constituyen un fundamento satisfactorio para distinguir entre almacenes separados. Vamos a considerar la validez de las pruebas en que se basa el modelo multialmacén con referencia a los conceptos de capacidad y codificación y finalmente de la propia función de retención.

\section{Capacidad}

Aunque la noción de la limitación de capacidad ha constituido un rasgo fundamental del enfoque en términos de flujo de información, y especialmente una característica del ACP en los modelos multialmacén, aún sigue siendo un tanto oscura la naturaleza exacta de esta limitación de capacidad. En concreto, no está claro si lo limitado es la capacidad de procesamiento, la de almacenamiento, o si se piensa que la limitación se aplica a alguna interacción entre ambas. Planteado en términos de la analogía del computador, en la que se basan los modelos del flujo de la información, el problema es el de si la limitación se refiere a la capacidad de almacenamiento de un registrador de memoria o a la velocidad con que el procesador puede efectuar ciertas operaciones. La noción de canal de capacidad limitada (Broadbent, 1958) parece hacer hincapié en la segunda interpretación, mientras que en los modelos posteriores de la memoria, como el de Waugh y Norman (1965) se favorece aparentemente la interpretación en términos de una limitación del almacenamiento. Miller (1956) hace las dos interpretaciones, pero no establece explícitamente la relación entre ellas.

Los intentos de medir la capacidad del ACP se han inclinado hacia la interpretación en términos de almacenamiento y han considerado que el número de items constituye una escala de medida apropiada. Estos intentos han proporcionado toda una gama de valores. Por ejemplo, las estima'ciones más recientes del tamaño de la memoria primaria (Baddeley, 1970; Mur- dock, 1972) han brindado valores que oscilan entre dos y cuatro palabras. Sin embargo, las medidas de la amplitud de memoria (que se dice que reflejan la capacidad limitada del almacén de memoria a corto plazo), se sitúan, característicamente, entre cinco y nueve items, dependiendo de que los items en cuestión sean palabras, letras o dígitos (Crannell y Parrich, 1957). Finalmente, cuando las palabras de una prueba de amplitud forman una oración, los sujetos (aún de menor edad) pueden reproducir cadenas de hasta 20 palabras (Craik y Masani, 1969). De modo que, si la capacidad constituye una característica crítica de la forma de operar de la MCP, el modelo en términos de «almacéns tiene que explicar por qué se dan esta gama tan amplia de estimaciones de la capacidad.

La explicación más aceptada de esta variación es la de que la capacidad está limitada en términos de «chunks» y que, dependiendo de la significación del material, podrán recodificarse pocos o muchos items en un achunki. Aparte de la dificultad de definir el «chunk» con independencia de sus consecuencias sobre la memoria, este punto de vista implica una concepción bastante flexible de la MCP como un compartimento de almacenamiento que puede aceptar una gran variedad de códigos, que van de los rasgos físicos más simples a los semánticos más complejos.

Desde el punto de vista de este artículo, el concepto de capacidad debe entenderse en términos de una limitación de procesamiento. Pensamos que las limitaciones de almacenamiento son consecuencia directa de esta limitación más fundamental.

\section{Codificaciōn}

Trabajando con un material verbal, Conrad (1964) y Baddley (1966) proporcionaron una base plausible para distinguir entre ACP y ALP. Sacaron la conclusión de 


\section{Estudios}

que la información de la MCP se atiene a un código acústico, mientras que el código del ALP sería predominantemente semántico. Sin embargo, las investigaciones posteriores han difuminado esta distinción. En primer lugar, se ha demostrado que la codificación del ACP puede ser o bien acústica o articulatoria (Levy, 1971; Peterson y Johnson, 1971). En segundo lugar, en artículos recientes Kroll y sus colaboradores (Kroll et al., 1970) han demostrado que, incluso con material verbal, el ACP puede ser, en ocasiones, visual. Aparentemente, el ACP puede aceptar diversos tipos de códigos físicos.

¿Puede mantener también el ACP información semántica? La persistencia de pruebas contradictorias indica que o bien esta cuestión se ha formulado inapropiadamente o bien la respuesta depende del paradigma que se utiliza. Cuando se toman en consideración los paradigmas tradicionales del ACP la respuesta parece ser que ano» (Kintsch y Buschke, 1969; Craik y Levy, 1970), aunque Shulman $(1970,1972)$ ha presentado recientemente pruebas muy considerables a favor de la existencia de un ACP de carácter semántico. Aunque originalmente parecía que el tipo de codificación constituía una buena base para la distinción entre memoria a corto plazo y memoria a largo plazo, esta distinción ya no parece satisfactoria. Los defensores de la concepción de términos de almacenes múltiples argumentarían que el código del ACP es flexible, pero con esta postura eliminarían una de las características importantes que permiten distinguir entre uno y otro almacén.

Nosotros argüimos que la cuestión del código puede formularse más apropiadamente en términos de las exigencias de procesamiento impuestas por el paradigma experimental y por el material que hay que recordar. En ciertos paradigmas y con determinados materiales, el código acústico puede ser más adecuado o el único posible. En otras circunstancias, el procesamiento a nivel semántico puede ser, al mismo tiempo, posible y ventajoso.

\section{Caracteristicas del olvido}

Parece que una condición mínima para diferenciar los almacenes de memoria en términos de sus características en cuanto al olvido es la de que la función de retención sea invariante para diversos paradigmas y condiciones experimentales. Aunque esta invarianza no se ha comprobado rigurosamente, hay casos en que está claro que no se da. Vamos a poner dos ejemplos. Primero, en los modelos en términos de estados finitos de aprendizaje de pares asociados, el estado que suele identificarse con el ACP muestra características de olvido. que son diferentes de las que se establecen para el ACP en otros paradigmas (Kintsch, 1970, pág. 206). En el primer caso, la retención del ACP se mantiene aun cuando haya 20 items intervinientes, mientras que en los paradigmas de recuerdo libre y de prueba (Waugh y Norman, 1965) la información se pierde mucho más rápidamente. $Y$ un segundo ejemplo: la duración del trazo de la memoria, en el caso de estímulos visuales, parece depender del material y del paradigma. Según Neisser (1967), el icón permanece durante un segundo o menos. Posner (1969) y sus colaboradores han encontrado pruebas de persistencia del icón visual durante $1.5 \mathrm{se}$ gundos, mientras que otros estudios recientes de Murdock (1971), Phillips y Baddeley (1971) y Kroll et al (1971 han brindado estimaciones de 6,10 y 25 segundos, respectivamente. Las estimaciones son aún mayores en el caso de la memoria de reconocimiento de dibujos (Shepard, 1967; Haber, 1970). Reconocemos dibujos, caras, canciones y voces después de largos períodos de tiempo, luego está claro que tenemos una memoria a largo plazo de informaciones no verbales relativamente literales. De modo que resulta difícil estable- 
cer una línea divisoria clara entre una «memoria sensorial» y una memoria «gráficas 0 srepresentacionals.

Nosotros defenderemos que la retención depende de determinados aspectos del paradigma como el tiempo de estudio, la cantidad de material presentado y la forma de prueba, y también de que el sujeto haya desarrollado más o menos sistemas para analizar y enriquecer determinados tipos de estímulos; es decir, de la familiaridad, compatibilidad y significación del material.

Aunque creemos que la formulación multialmacén es insatisfactoria cuando se considera en términos de capacidad, codificación y características del olvido, es evidente que hay ciertos resultados básicos que debe incorporar cualquier modelo. Parece cierto que los estímulos se codifican de diversas maneras en el sistema de memoria: una palabra puede codificarse en distintos momentos en términos de sus rasgos visuales, fonémicos o semánticos, de sus asociaciones verbales o de una imagen. Parece que las representaciones codificadas de distintas maneras persisten durante períodos de tiempo diferentes. El fenómeno de la existencia de una limitación de la capacidad en ciertos puntos del sistema parece suficientemente real, por lo que debe tomarse en consideración. Finalmente, habría que señalar también el papel de los procesos perceptivos, atencionales y de repaso.

Una forma de enfrentarse con las inconsistencias del tipo de las que hemos descrito consiste en proponer más almacenes (ver Morton, 1970; Sperling, 1970). Sin embargo, nosotros pensamos que resulta más útil dirigir la atención a las propias operaciones de codificación y tomar en consideración la propuesta de que las tasas del olvido están en función del tipo de codificación y de la profundidad de ésta. Este punto de vista es el que se desarrolla en la sección siguiente.

\section{NIVELES DE PROCESAMIENTO}

Muchas teorías actuales concuerdan en que la percepción implica un análisis rápido de los estímulos a varios niveles o estadios (Selridge y Neisser, 1960; Treisman, 1964; Sutherland, 1968). En los primeros estadios se realiza el análisis de determinadas características físicas o sensoriales como las líneas, los ángulos, el brillo, el tono y la sonoridad, mientras que los estdios finales se relacionan más con la comparación entre input y las abstracciones almacenadas a partir del aprendizaje pasado. Es decir: en los últimos estadios se realiza el reconocimiento de patrones y la extracción del significado. Solemos referirnos a esta concepción de una serie o jerarquía de estadios de procesamiento hablando de «profundidad de procesamiento», en que una mayor "profundidad» implica un mayor grado de análisis semántico o cognitivo. Después de que el estímulo ha sido reconocido, puede procesarse más por enriquecimiento o asociación. Por ejemplo, después de que una palabra ha sido reconocida, ésta puede evocar asociaciones, imágenes o historias basadas en la experiencia pasada del sujeto con esa palabra. Esa «codificación por elaboración (Tulving y Madigan, 1970) no se limita al material verbal. Nosotros defendemos que en el análisis perceptivo de los sonidos, datos visuales, olores, etc., se dan niveles similares de procesamiento. El análisis procede a traves de una serie de estadios sensoriales, hasta los niveles relacionados con la comparación o reconocimiento del estímulo y, finalmente, hasta los estadios semántico-asociativos de enriquecimiento del estímulo.

Uno de los resultados de este análisis perceptivo es el trazo de la memoria. Así, ciertos rasgos de éste, como sus características de codificación y su persistencia, surgen esencialmente como subproductos del procesamiento perceptivo (Morton, 1970). Específicamente sugerimos que la persis- 
tencia del trazo es una función de la profundidad del análisis, y los niveles más profundos de análisis se asocian con trazos. más elaborados, más persistentes y más intensos. Como normalmente al organismo le preocupa sólo la extracción del significado de los estímulos, resulta ventajoso almacenar los productos de ese análisis profundo, pero normalmente no hay necesidad de almacenar los productos de los análisis preliminares. Es perfectamente posible dibujar un cuadro alrededor de estos primeros análisis y llamarlos «memoria sensorial» y otro alrededor de los análisis intermedios denominándolo «memoria a corto plazo», pero con este procedimiento simplificamos excesivamente las cosas y evadimos los problemas más significativos.

Aunque ciertas operaciones analíticas deben preceder a otras, muchas pruebas recientes indican que percibimos a un nivel significativo más profundo, antes de percibir los resultados de otros análisis lógicamente previos (MacNamara, 1972; Savin y Bever, 1970). No es que se dé una elaboración del código en una jerarquía invariante de pasos, y ello parece especialmente cierto en lo que se refiere a los estadios últimos de procesamiento. En este sentido describiríamos mejor las cosas hablando de «amplitud» de codificación, pero mantendremos el término "profundidad», dado que transmite el sentido de nuestra argumentación.

Los estímulos muy familiares y significativos son compatibles, por definición, con las estructuras cognitivas existentes. Tales estímulos (por ejemplo, los dibujos y oraciones) se procesarán a un nivel profundo más rápidamente que los estímulos menos significativos, y se retendrán mejor que éstos. Así, la rapidez del análisis no predice necesariamente la retención. La retención está en función de la profundidad y hay varios factores que determinan la profundidad con que se procesa un estímulo, entre ellos la cantidad de atención que se le dedica, su compatibilidad con las es- tructuras analizadoras y el tiempo disponible para el procesamiento.

Por tanto, nosotros preferimos concebir la memoria como vinculada a los niveles de procesamiento perceptivo. Aunque estos niveles puedan agruparse en estadios (análisis sensorial, reconocimiento de patrones y elaboración del estímulo, por ejemplo) es más útil concebir los niveles de procesamiento como un continuo de análisis. Así, la memoria también se considera como un continuo que va de los productos transitorios de los análisis sensoriales a los muy duraderos de las operaciones semántico-asociativas. Sin embargo, superpuesto a este sistema básico de memoria hay otra forma posible de retener los estímulos: haciendo circular la información, una y otra vez, a un cierto nivel de procesamiento. Según nuestro punto de vista, ciertas descripciones como «atención continuada a ciertos aspectos del estímulo», «mantener los items en la conciencia», «retener los items en el «rehearsal buffer» y «retención de los items en la memoria primarias se refieren todos ellos al mismo concepto de mantener la información a un cierto nivel de procesamiento. Para mantener una cierta continuidad con la terminología existente, utilizaremos el término «memoria primaria» (M.P.) para referirnos a esta operación, aunque hay que señalar que nuestro uso del término es más restringido de lo que suele serlo.

Apoyamos la noción de Moray (1967) de que hay un procesador central de capacidad limitada que puede emplearse de varias formas distintas. Si esta capacidad de procesamiento se utiliza para mantener la información a un solo nivel, aparecerían los fenómenos de la memoria a corro plazo. El propio procesador es neutral con respecto a las características de codificación: el código observado de M.P. dependerá de la modalidad de procesamiento con la que opera el procesador. Además, aunque la capacidad limitada está en función del propio procesador, el número de items mantenidos dependerá del nivel al 
que está operando. A niveles más profundos el sujeto puede utilizar más las reglas aprendidas y el conocimiento pasado; así podrá manejar más eficientemente el material y retenerlo mejor. Aparentemente es muy variable la facilidad con que puede mantenerse la información en la MP a diversos niveles. Parece que ciertos tipos de información (por ejemplo, los rasgos fonémicos de las palabras) son particularmente fáciles de mantener, mientras que otros (como los análisis visuales preliminares, el «icón») son aparentemente imposibles.

El rasgo esencial de la retención de la MP es el hecho de que aún se están procesando o atendiendo ciertos aspectos del material. De modo que nuestra noción de la MP es sinónima a la de James (1890), puesto que los items de la MP se mantienen aún en la conciencia. Cuando se deja de prestar atención al item, la información se pierde a la tasa propia de su nivel de procesamiento, las tasas serán más lentas cuando los niveles de análisis han sido más profundos.

La retención de la memoria es, por tanto, equivalente a un procesamiento continuado, pero este tipo de procesamiento simplemente prolonga la alta accesibilidad de un determinado item sin llevar, por ello, a la formación de un trazo de memoria más permanente. Este procesamiento de tipo I (es decir, la repetición de los análisis que ya se han llevado a cabo) puede continuarse con el procesamiento de tipo II, que implica un análisis más profundo del estímulo. Sólo este segundo tipo de repaso producirá un aumento de la ejecución de la memoria. En tanto en cuanto el sujeto utilice un procesamiento de tipo II, su memoria mejorará a medida que aumente su tiempo total de estudio, pero cuando realiza un procesamiento de tipo I deja de ser aplicable la «hipótesis del tiempo total (ver Cooper y Panite, 1967). Stoff y Eagle (1971) han recogido hallazgos que son acordes con esta sugerencia.
En resumen, sugerimos que el trazo de la memoria se describe mejor en términos de la profundidad de procesamiento o grado de elaboración del estímulo. Los análisis más profundos producen trazos más persistentes. Aunque la información pueda mantenerse en la MP, ese mantenimiento no mejora, por sí mismo, la retención subsiguiente; cuando se deja de prestar atención la información se pierde a una tasa que depende esencialmente del nivel de análisis.

\section{REEXAMEN DE LOS DATOS EXISTENTES}

\section{Aprendizaje incidental}

Cuando los trazos de la memoria se consideran un producto de una cierta forma de procesamiento, gran parte de la literatura sobre aprendizaje incidental adquiere una nueva significación. Hay varias revisiones de esta literatura (Postman, 1964; McLaughlin, 1965) y no trataremos de ser exhaustivos. Una característica importante del paradigma de aprendizaje incidental es que el sujeto procesa el material de una forma compatible con la tarea orientadora, o que está determinada por ésta. Por eso, la comparación de la retención en distintas tareas orientadoras proporciona una medida relativamente pura de las consecuencias, en la memoria, de diversas actividades de procesamiento.

Según el punto de vista que mantenemos en este artículo, y de acuerdo con Postman (1964), la instrucción explícita de que aprendan un material facilita la ejecución de los sujetos sólo cuando les lleva a procesar el material de forma más efectiva que la que tuvieran en la situación incidental, dependiendo de las instrucciones de orientación. Así, el aprendizaje en condiciones incidentales puede ser superior al que se da en condiciones intencionales, cuando la tarea de orientación es apropiada y la estrategia intencional inapropiada. 
Desde el punto de vista de este artículo, entonces lo que resultaría interesante sería estudiar sistemáticamente la retención consecuente a diversas tareas de orientación, más que comparar el aprendizaje incidental con el intencional. En condiciones incidentales, el experimentador tiene control sobre el procesamiento que el sujeto aplica al material, cosa que no ocurre cuando nos limitamos a instruir al sujeto para que aprenda y éste utiliza una estrategia de codificación desconocida.

Vamos a considerar varios ejemplos que ilustran este punto. Tresselt y Mayzner (1960) probaron el recuerdo libre después de un aprendizaje incidental, con tres tareas de orientación diferentes: tachar vocales, copiar las palabras y juzgar hasta qué punto era cada palabra un ejemplo del concepto «económico». En la última condición, el número de palabras recordadas era cuatro veces mayor que en la primera y dos veces mayor que en la segunda. Hyde y Jenkins (1969) y Johnston y Jenkins (1971) han obtenido resultados similares, utilizando el paradigma de recuerdo libre. Los experimentos de Jenkins y sus colaboradores demostraron que con listas de pares de palabras muy asociadas, el recuerdo libre y la organización resultante de una tarea de orientación que requería el empleo de las palabras como unidades semánticas era equivalente al de un grupo de control intencional cuyos sujetos no recibían ninguna tarea incidental, pero, en cambio, eran relativamente superiores a un grupo que realizaba un aprendizaje incidental y cuya tarea suponía tratar la palabra estructuralmente (comprobar. si había ciertas letras o estimar el número de letras de cada palabra). Estos resultados son consistentes con los de Mandler (1967), que demostró que el aprendizaje incidental durante la categorización de unas palabras provocaba un nivel semejante de recuerdo que el de un grupo que realizaba la misma actividad, pero cuyos miembros sabían que se iba a comprobar su memoria.
Los experimentos en que se ha estudiado el aprendizaje incidental de oraciones (Bobrow y Bower, 1919; Rosemberg y Schiller, 1971 han demostrado que el recuerdo después de una tarea de orientación que exigía el procesamiento de la oración a un nivel semántico era substancialmente superior al recuerdo de palabras de oraciones expuestas de forma equivalente, pero que no se procesaban semánticamente.

Schulmam (1971) hizo a sus sujetos que buscaran en una lista ciertas palabras clave definidas estructuralmente (por ejemplo, palabras con la letra A) o semánticamente (por ejemplo, palabras que denotaran algo vivo). Después de realizar esta tarea de búsqueda, se pasaban a los sujetos una prueba inesperada de memoria de reconocimiento. La ejecución era significativamente superior en las condiciones en que la clave se definía semánticamente que en aquellás en que se definía estructuralmente, aunque el tiempo de trabajo con cada palabra era aproximadamente el mismo en uno y otro caso.

Estos resultados apoyan la conclusión general de que el rendimiento de la memoria constituye una función positiva del nivel de procesamiento exigido por la tarea de orientación. Sin embargo, más allá de un cierto estadio, la forma de procesamiento óptima dependerá de las exigencias de recuperación o de utilización de trazo en la prueba subsiguiente de memoria. Existen pruebas claras, en la literatura sobre aprendizaje incidental, que demuestran que el valor relativo de las distintas tareas de orientación no es el mismo para todas las pruebas de memoria.

Esta conclusión se ve apoyada cuando se comparan los distintos efectos de las tareas de orientación en el reconocimiento y el recuerdo. Eagle y Leitèr (1964) encontraron que el recuerdo libre en una situación de aprendizaje intencional sin estorbos era superior al de un grupo incidental y al de otro grupo intencional en que los sujetos también tenían que realizar su tarea de 
orientación; sin embargo, en estas dos situaciones el rendimiento en una prueba de reconocimiento era superior. Este resultado no plantea dificultades si suponemos que el procesamiento óptimo no es el mismo un una y otra prueba de memoria. En el experimento de Eagler y Leiter (1964) la tarea de orientación implicaba, casi con seguridad, un cierto grado de análisis semántico, pero podría haber servido para impedir el procesamiento de elaboración necesario para acceder posteriormente a la información almacenada. Por otra parte, esa codificación de elaboración podría estorbar a la discriminación posterior entre las palabras clave y los distractores relacionados asociativamente, que se utilizaron en ese experimento. Dornbush y Winnick (1967) y Estes y Da Polito (1967) han encontrado resultados que son coherentes con este tipo de análisis.

Todas las tareas de orientación utilizadas por Wicker y Bernstein (1969) en su estudio sobre aprendizaje incidental de pares asociados requerían un análisis a nivel semántico, pero no facilitaban la ejecución subsiguiente al mismo nivel. Cuando la tarea de orientación exigía la producción de respuestas mediadoras, la ejecución era igual que la que se daba en el aprendizaje intencional sin estorbos y superior a la que se daba cuando la tarea de orientación consistía en evaluar lo agradables que resultaban las palabras. En el recuerdo libre de un solo ensayo, esta última tarea de orientación produce una ejecución igual a la que se da en el aprendizaje intencional (Hyde y Jenkins, 1969). Las mismas tareas de orientación no parecen tener efectos equivalentes en distintos paradigmas. Es importante acentuar la existencia de una interacción entre la codificación inicial y las operaciones subsiguientes de recuerdo. Aunque la distinción entre la disponibilidad y accesibilidad (Tulving y Pearlstone, 1966) es útil, la efectividad de una señal de recuperación depende de su compatibilidad con la codificación inicial del item o, más en gene- ral, de hasta qué punto la situación de recuperación reinstala el contexto de aprendizaje.

\section{Atención selectiva y almacén sensorial}

Moray (1969) demostró que las palabras presentadas por el canal no atendido en una prueba de escucha dicótica no eran reconocidas en una prueba posterior de memoria. De forma similar, Neisser (1964) ha demostrado que los items que no servían de clave en una tarea de búsqueda visual no dejaban efectos recognoscibles. De forma que cuando los estímulos sólo se analizan parcialmente, o se procesan sólo a niveles periféricos su registro en la memoria es extremadamente fugaz. Treismem (1964) lo demostró claramente. Cuando se presentaban a ambos oídos - dicóticamente- un mismo párrafo de prosa, de tal manera que había una diferencia temporal entre el momento en que los mismos sonidos llegaban a uno y otro oído y era anterior el del oído no atendido, la diferencia temporal entre uno y otro mensaje tenía que reducirse hasta 1.5 segundos para que los sujetos se diesen cuenta de que los dos mensajes eran idénticos. Sin embargo, cuando era anterior el mensaje del oído atendido (sombreado) los sujetos notaban la semejanza con un retraso medio de 4.5 segundos entre uno y otro mensaje. Así, aunque los sujetos no trataban de recordar el material en ninguno de los casos, el hecho de que el procesamiento exigido por el sombreado fuera mayor era suficiente para triplicar la duración del trazo de memoria. Treisman también encontró que la significación del material (habla invertida v.s. habla normal y palabras al azar v.s. prosa) afectaba al intervalo temporal necesario para el reconocimiento, pero sólo cuando el canal atendido era el primero por el que se transmitía el mensaje. Cuando el mensaje era rechazado después de los primeros análisis, la significación no jugaba ningún papel, pero cuan- 
do se atendía al mensaje los materiales significativos podían procesarse más y, por tanto, podían retenerse durante más tiem. po. Las tres estimaciones de la persistencia de la memoria en estos experimentos (1.5 segundos para el material no atendido, 3 segundos para el habla invertida atendida y las cadenas atendidas de palabras al azar y 5 segundos para la prosa atendida) pueden atribuirse al funcionamiento de almacenes diferentes, pero es más razonable, según nuestro punto de vista, proponer que la persistencia está en función del nivel de procesamiento.

No vamos a revisar más estudios con el mismo detalle, pero debemos señalar que los resultados y las conclusiones de muchos otros investigadores en el área de la memoria sensorial pueden acomodarse también a este cuadro. Neisser (1967, página 33) concluía que «las exposiciones más largas producen icones más perdurables». Los estudios de Norman (1969), Glucksberg y Cowen (1970) y Peterson y Kroener (1964) pueden interpretarse en el sentido de que demuestran que el material verbal no atendido se pierde en pocos segundos.

Massaro (1970) sugirió que la memoria de un item se relaciona directamente con la cantidad de procesamiento perceptivo de dicho item, afirmación que se sitúa, evidentemente, en la misma línea que las propuestas aquí presentadas, si bien sus argumentos posteriores (Massaro, 1970) según los cuales la memoria ecoica dura, inevitablemente, sólo 250 milisegundos, son probablemente generalizaciones excesivas. A partir de un experimento de reconocimiento de dibujos, Shaffer y Shiffrin sacaron la conclusión de que «quizá sea más fructífero considerar que el punto de vista más acorde con la parsimonia es el de que sólo hay una memoria visual a corto plazo. Esta memoria visual a corto plazo decae rápidamente cuando el contenido informativo del campo visual es alto y más despacio cuando se reduce mucho el contenido informativo» (Shaffer y Shiffrin,
1972, pág. 295). En lo fundamental, este punto de vista es similar al nuestro, aunque nosotros añadiríamos que ese continuo abarca también a la retención a largo plazo. A ello añadiríamos que lo que determina la tasa de desvanecimiento del trazo es el nivel de procesamiento, más que el contenido informativo.

\section{La distinciōn $A C P / A L P$}

El fenómeno de un mecanismo de mantenimiento de capacidad limitada en la memoria (Miller, 1956; Broadbent, 1958) se sitúa en este cuadro conceptual suponiendo que hay un procesador central flexible que puede desplegarse en uno de entre varios niveles y con arreglo a una dimensión de codificación entre varias, y que este procesador central sólo puede manejar un número limitado de items en un determinado momento. Es decir, los items se mantienen en la conciencia o en la memoria primaria manteniendo su repaso a un cierto nivel fijo de procesamiento. La naturaleza de los items dependerá de la dimensión de codificación y del nivel en dicha dimensión. A niveles más profundos el sujeto puede hacer más uso de las estructuras cognitivas aprendidas, de modo que el item se irá haciendo más complejo y semántico. La profundidad con la que opera la memoria primaria dependerá de la utilidad que tenga para el sujeto el seguir procesando a ese nivel y también de lo susceptible que sea el material de un procesamiento más profundo. Así, si la tarea del sujeto consiste simplemente en reproducir pocas palabras unos segundos después de leerlas, no necesita mantenerlas a un nivel más profundo que el del análisis fonémico. Pero si las palabras constituyen una oración significativa, entonces son compatibles con estructuras aprendidas más profundas y pueden manejarse unidades mayores. Parece que la memoria primaria maneja, en cualquier nivel, unidades o «paquetes» más que información (ver Kintsch, 
1970, págs. 175-181). Es decir, repasamos un sonido, una letra, una palabra, una idea o una imagen del mismo modo que percibimos objetos y no constelaciones de atributos.

- Como señalábamos antes, una distinción que suele hacerse entre los almacenes de memoria es la que se basa en sus diferentes características de codificación; se dice que el ACP es predominantemente acústico (o articulatorio), mientras que el ALP es, sobre todo, semántico. Según la argumentación que aquí mantenemos, los errores acústicos sólo serán predomiantes en tanto en cuanto no se haya llevado a cabo el análisis a un nivel semántico. Hay al menos tres factores que pueden influir en que el procesamiento no alcance ese nivel: la naturaleza del material, la limitación de la capacidad de procesamiento disponible y las exigencias de la tarea. Muchos de los datos sobre las confusiones acústicas en la memoria a corto plazo se basan en materiales como letras y dígitos que tienen relativamente poco contenido semántico. Estos materiales, por su propia naturaleza, tienden a limitar el procesamiento a un nivel estructural de análisis, por lo no resulta sorprendente que den lugar a errores de naturaleza estructural. Estos errores pueden darse también con los materiales significativos cuando la capacidad de procesamiento se desvía a una tarea irrelevante (Eagle y Ortoff, 1967).

Otra serie de resultados relevantes para la distinción entre ACP y ALP es la de los que demuestran que en el recuerdo libre las variables como la tasa de presentación y la frecuencia de las palabras afectan a la retención a largo plazo, pero no a la retención a corto plazo (Glanzer, 1972). Nuestra interpretación de estos resultados es que, al aumentar la tasa de presentación o utilizar palabras que no son familiares, se inhibe o impide el procesamiento que se realiza a los niveles necesarios para apoyar la retención a largo plazo, pero ello no afecta a las operaciones de codificación del tipo de las que son adecuadas para la retención a corto plazo. De esta interpretación se sigue que el hecho de desviar la capacidad de procesamiento, como se hace en los experimentos de Eagle y Ortoff (1967) deberá dar como resultado un decremento mayor de la retención a largo plazo que de la retención a corto plazo y, en realidad existen pruebas que demuestran que eso es lo que ocurre (Murdock, 1965; Silverstein y Glanzer, 1971).

Por el contrario, las manipulaciones que influyen en el procesamiento a un nivel estructural tendrán efectos transitorios, pero no efectos a largo plazo. Las diferencias de modalidad (Murdock, 1966) proporcionan un ejemplo claro. Finalmente, las manipulaciones que induzcan un procesamiento más profundo o más elaborado deberán facilitar el recuerdo a largo plazo. Pensamos que la hipótesis de la variabilidad de codificación, tal como se ha utilizado para explicar el efecto de espaciación en el recuerdo libre (Madigan, 1965; Melton, 1970), debe entenderse en estos términos.

\section{La curva de posición serial}

Los efectos de posición serial han constituido una fuente de evidencia fundamental para establecer la distinción entre ACP y ALP (ver Broadbent, 1971, págs. 354-361; Kintsch, 1970, págs. 53-162). En el recuerdo libre se supone que el efecto de recencia refleja el «out-put» del ACP mientras que los items previos se recuperan del ALP (Glanzer y Cunitz, 1966). Se han propuesto varias explicaciones teóricas del efecto de primacía, pero quizá la más plausible sea la de que los items iniciales reciben más repaso, por lo que se registran mejor en el ALP (Atkinson y Shiffrin, 1968; Bruce y Papay, 1970). Nosotros estamos de acuerdo con estas conclusiones. Como el sujeto sabe que debe dejar de atender a los items iniciales para percibir y reciclar los posteriores, somete a esos items a un procesamiento de tipo II; es decir, a 
un procesamiento semántico más profundo. Sin embargo, los items finales de la lista pueden sobrevivir con una codificación fonémica, que da lugar a un recuerdo inmediato excelente (puesto que aún se están procesando en la memoria primaria), pero éste se borra por la necesidad de procesar material interpolado. De hecho, si los items finales se han procesado con menos profundidad que los iniciales, la formulación en términos de niveles de procesamiento predeciría que en un intento posterior de recuerdo, los items finales serán los que menos se recuerdan de toda la lista. El descubrimiento de la recencia negativa (Craik, 1970) apoya esta predicción. Una explicación alternativa de la recencia negativa es la de que los items recientes se recuerdan porque se repasaron menos veces que los primeros (Rundus, 1971). Sin embargo, los estudios recientes de Jacoby y Bartz (1972), Watkins (1972) y Craik (1972) han demostrado que lo que determina el recuerdo subsiguiente de los últimos items de una lista es el tipo de procesamiento más que la cantidad de éste.

En el aprendizaje serial, los sujetos deben retener los primeros items para poder por lo menos, comenzar correctamente su recuerdo. El gran aumento del efecto de primacía es, entonces, atribuible - por lo menos en parte- a la retención en la memoria primaria. Además, el que los sujetos también codifiquen los items iniciales a un nivel más profundo depende, probablemente, del material y de la tarea. Utilizando un ritmo de presentación relativamente lento (2.5 segundos) y empleando palabras como estímulos, con presentación visual, Palmer y Ornstein (1971) encontraron que el hecho de interpolar una tarea sólo eliminaba parcialmente el efecto de primacía. Sin embargo, Baddeley (1968) presentó auditivamente dígitos a un ritmo de 1 por segundo y encontró que se eliminaba completamente el efecto de primacía debido a la necesidad de efectuar otra tarea.

\section{Efectos de repetición y de repaso}

Una sugerencia que se deduce de esta formulación es que el procesamiento de tipo I no contribuye a incrementar la memoria del estímulo: cuando la atención se dirige a otra cosa, el trazo se pierde al ritmo propio del nivel más profundo al que se ha analizado. Así, el concepto de procesamiento se ha dividido en dos: un procesamiento de tipo I, o del mismo nivel y un procesamiento de tipo II que implica un análisis posterior, más profundo, del estímulo y produce un trazo más duradero. De forma similar, los efectos de la repetición de la presentación dependen de que el estímulo repetido sea simplemente procesado al mismo nivel o se codifique de forma diferente en las presentaciones posteriores. Existen pruebas de que la repetición de un item codificado a un solo nivel sensorial, ya sea la audición (Moray, 1959; Norman, 1969) o la visión (Turvey, 1967) no produce un incremento del rendimiento de la memoria.

Tulving (1967) ha demostrado también que la repetición sin intención de apren. der no facilita el aprendizaje. La explicación que da Tulving de este hecho en términos de la organización interitems no puede diferenciarse claramente de una explicación en términos de niveles de procesamiento. De forma similar, Glanzer y Meinzer (1967) han demostrado que la repetición explícita de los items en el recuerdo libre es una estrategia menos efectiva que la que normalmente utilizan los sujetos. Aunque tanto Waugh y Norman (1965), como Atkinson y Shiffrin (1968), han sugerido que el repaso tiene la doble función de mantener la información en la memoria primaria y transferirla a la memoria secundaria, los experimentos de Tulving (1966) y Glanzer y Meinzer (1967) demostraron que ello no tiene por qué ser así necesariamente. Así, el que el repaso fortalezca el trazo o simplemente pospon- 
ga el olvido depende de lo que el sujeto haga con su repaso. Sólo el aumento de profundidad del procesamiento producirá un incremento de la memoria.

\section{COMENTARIOS FINALES}

Nuestra explicación de la memoria en términos de niveles de procesamiento tiene muchas cosas en común con otras varias formulaciones recientes. Cermak (1971), por ejemplo, ha bosquejado un marco teórico muy similar al nuestro. Las teorías de la codificación de atributos, de orientación perceptiva, como las de Bower (1967) y Norman y Rumelhart (1970) son muy afines a este enfoque, como lo es la de Posner (1969), que propone la existencia de estadios de procesamientos y asocia características diferentes a cada estadio.

Si se considera que el trazo de la memoria constituye un subproducto del análisis perceptivo, un objetivo importante de la investigación futura será el de especificar las consecuencias que tienen sobre la memoria los distintos tipos de operaciones perceptivas. Ya hemos sugerido que la comparación entre distintas tareas de orientación en el paradigma de aprendizaje incidental, constituye un método con el que el experimentador puede tener un control más directo sobre las operaciones de codificación que efectúan los sujetos. Como los análisis más profundos suelen implicar un tiempo más dilatado de procesamiento, sería muy importante discriminar entre la influencia de variables como el tiempo de procesamiento y la cantidad de esfuerzo y la de la profundidad como tal. Por ejemplo, el tiempo puede ser un correlato de la memoria en tanto en cuanto sea necesario para procesar a un cierto nivel, pero es posible que el tiempo «de más» que se dedica a un simple repaso de la información, después de alcanzar ésta su nivel óptimo, no prediga la perdurabilidad del trazo.

Nuestro enfoque no constituye una teoría de la memoria. Más bien proporciona un marco conceptual - una serie de acticudes orientadoras - con arreglo al cual podría proceder la investigación sobre la memoria. Aunque los modelos en términos de almacenes múltiples han ejercido una función útil, creemos que muchas veces se han tomado en un sentido demasiado literal y que esta formulación que presentamos se presta a formularse preguntas más fructíferas. Evidentemente, nuestra posición es especulativa y no está completa, ni mucho menos. Hemos considerado la memoria sólo desde el punto de vista del «input» o polo de la codificación; no hemos tratado de especificar cómo se diferencian unos items de otros, cómo se agrupan y organizan ni cómo se recuperan del sistema. Aunque nuestra posición no presupone ningún enfoque específico de estos procesos, proporciona un marco apropiado para su comprensión.

\section{Referencias}

ATKINSON, R. C., y SHIFRIN, R. M. Human memory: A proposed system and its control processes. En K. W. Spence y J. T. Spence (eds.). The psychology of learning and motivation: Advances in research and theory, Vol. II. Nueva York. Academic Press, 1968, págs. 89-195.

ATKINSON, R. C., y SHIFFRIN, R. M. The control of short-term memory. Scientific American, $1971,224,82-89$.

BADDELEY, A. D. Short-term memory for word sequences as a function of acoustic, semantic, and formal similarity. Quarterly Journal of Experimental Psychology, 1966, 18, 362-365. 
BADDELEY, A. D. How does acoustic similarity influence short-term memory? Quarterly Journal of Experimental Psychology, 1968, 20, 249-264.

BADDELEY, A. D. Estimating the short-term component in free recall. British Journal of Psychology, 1970, 61, 13-15.

BOBROW, S. A., y BOWER, G. H. Comprehension and recall of sentences. Journal of Experimental Psychology, 1969, 80, 455-461.

BOWER, G. H. A multicomponent theory of the memory trace. En K. W. Spence Y J. T. Spence (eds.). The psychology of learning and motivation: Advances in research and theory, Vol. 1. Nueva York. Academic Press, 1967, págs. 230-325.

BROADBENT, D. E. Perception and communication. Nueva York. Pergamon Press, 1958.

BROADBENT, D. E. Decision and stress. Nueva York. Academic Press, 1971.

BRUCE, D., y PAPAY, J. P. Primacy effect in single-trial free recall. Journal of Verbal Learning and Verbal Behavior, 1970, 9, 473-486.

CERMAK, L. S. Human memory. Research and theory. Nueva York. Ronald, 1972.

CONRAD, R. Acoustic confusions in immediate memory. British Journal of Psychology, 1964, 55, 75-84.

COOPER, E. H., y PANTLE, A. J. The total-time hypothesis in verbal learning. Psychological Bulletin, 1967, 68, 221-234.

CRAIK, F. J. M. The fate of primary memory items in free recall. Journal of Verbal Learning and Verbal Behavior, 1970, 9, 143-148.

CRAIK, F. J. M. A alevels of analysis» view of memory. Comunicación presentada en el $2 .^{\circ} \mathrm{Sim}$ posium de Erlindale sobre Comunicación y Afecto, marzo 1972.

CRAIK, F. L. M., Y LEVY, B. A. Semantie and acoustic information in primary memory. Journal of Experimental Psychology, 1970, 86, 77-82.

CRAIK, F. L. M., Y MASANI, P. A. Age and intelligence differences in coding and retrieval of word lists. British Journal of Psychology, 1969, 60, 315-319.

CRANNELL, C. W., Y PARRISH, J. M. A comparison of immediate memory span for digits, letters, and words. Journal of Psychology, 1957, 44, 319-327.

CROWDER, R. G., Y MORTON, J. Precategorical acoustic storage. Perception and Psychophysics, 1969, 5, 365-373.

DORNBUSH, R. L., y WINNICK, W. A. Short-term intentional and incidental learning. Journal of Experimental Psychology, 1967, 73, 608-611.

EAGLE, M., y LETTER, E. Recall and recognition in intentional and incidental learning. Journal of Experimental Psychology, 1964, 68, 58-63.

EAGE, M., y ORTOFF, E. The effect of level of attention upon sphonetic recognition errors. Journal of Verbal Learning and Verbal Behavior, 1967. 6, 226-231.

ESTES, W. K., y DAPOLITO, F. Independent variation of information storage and retrieval processes in paired-associate learning. Journal of Experimental Psychology, 1967, 75, 18-26.

GLANZER, M. Storage mechanisms in recall. En G. H. Bower (ed.). The psychology of learning and motivation: Advances in research and theory. Vol. 5. Nueva York. Academic Press, 1972, págs. 129-193.

GLANZER, M., y CUNITZ, A. R. Two storage mechanisms in free recall. Journal of Verbal Learning and Verbal Behavior, 1966, 5, 351-360.

GLANZER, M., y MEINZER, A. The effects of intralist activity on tree recall. Journal of Verbal Lecirning and Verbal Behavior, 1967, 6, 928-935.

GLUCKSBERG, S., y COWEN, G. N. Memory for nonattended auditory material. Cognitive Psychology, 1970, 1, 149-156.

HBER, R. N. How we remember what we see: Scientific American, 1970, 222, 104-112.

HYDE, T. S., Y JENKINSS, J. J. The differential effects et incidental tasks on the organization of recall of a list of highly associated words. Journal of Experimental Psychology, 1969, 82, 472-481.

JACOBY, L. L., y BARTZ, W. H. Encoding processes and the negative recency effect. Journol of Verbal Learning and-Verbal Behavior, 1972, 11, 561-565.

JAMES, W. Principles of psychology. Nueva York. Holt, 1890.

JOHNSTON, C. D., y JENKINS, J. J. Two more incidental tasks that differentially affect associative clustering in recall. Journal of Experimental Psychology, 1971, 89, 92-95.

KINTSCH, W. Learning, memory, and conceptual processes. Nueva York, 1970.

KINTSCH, W., y BUSCHKE, H. Homophones and synonyms in short-term memory. Journal of Experimental Psychology, 1969, 80, 403-407.

KROLL, N. E. A.; PARKS, T.; PARKINSSON, S. R.; BIEBER, S. L., y JOHNSON; A. L. Short-term memory while shadowing. Recall of visually and aurally presented letters. Journal of Experimental Psychology, 1970, 85, 220-224.

LEVY, B. A. Role of articulation in auditory and visual short-term memory. Journal of Verbal Learning and Verbal Behavior, 1971, 10, 123-132.

MACNAMARA, J. Cognitive basis of language learning in infants. Psychological Review, 1972. $79,1-13$. 
MADIGAN, S. A. Intraserial repetition and coding processes in free recall. Journal of Verbal Learning and Verbal Behavior, 1969, 8, 828-835.

MANDLER, G. Organization and Memory. En K. W. Spence and J. T. Spence (eds.). The psychology of learning and mutivation: Advances in research and theory, Vol. l. Nueva York. Academic Press, 1967, págs. 328-372.

MASSARO, D. W. Preperceptual images, processing time, and perceptual units in auditory percep1970, 77, 557-567.

MASSARO, D. W. Perceptual images, processing time, and perceptual unita in auditory perception. Psychological Review, 1972, 79, 124-145.

MCLAUGHLIN, B. "Intentional" and "incidental learning in human subjects: The role of instructions to learn and motivation. Psychological Bulletin, 1965, 63, 359-376.

MELTON, A. W. Implications of short-term memory for a general theory of memory. Journal of Verbal Learning and Verbal Behavior, 1963, 2, 1-21.

MELTON, A. W. The situation with respect to the spacing of repetitions and memory. Journal of Verbal Leorning and Verbal Behovior, 1970, 9, 596-606.

MILLER. G. A. The magical number seven, plus or minus two: Some limits on our capacity for processing information. Psychological Review, 1956, 63, 81-97.

MILNER, B. Memory and the medial temporal regions of the brain. En K. H. Pribram and D. E. Broadbent (eds.). Biology of memory. Nueva York. Academic Press, 1970, págs. 29-50.

MORAY, N. Attention in dichotic listening: affective cues and the influence of instructions. Quarterly Journal of Experimental Psychology, 1959, 9, 56-60.

MORAY, N. Where is capacity limited? A survey and a model. En A. Sanders (ed.). Attention and performance. Amsterdam. North-Holland, 1967.

MORTON: J. A. functional model of memory. En D. A. Norman (ed.). Models of human memory. Nueva York. Academic Press, 1970, págs. 203-254.

MURDOCK, B. B. JR. Effects of a subsidiary task on short-term memory. British Journal of Psychology, 1965, 56, 413-419.

MURDOCK, B. B. JR. Visual and auditory stores in short-term memory. Quarterly Journal of Experimental Psychology, 1966, 18, 206-211.

MURDOCK, B. B. JR. Recent developments in short-term memory. British Journal of Psychology, $1967,58,421-433$.

MURDOCK, B. B. JR. Four channel effects in short-term memory. Psychonomic Science, 1971, 24, 197-198.

MURDOCK, B. B. JR. Short-term memory. En G. H. Bower (ed.), Psychology of learning and motivation. Vol. 5. Nueva York. Academic Press, 1972, págs. 67-127.

NEISSER, U. Visual search. Scientific American, 1964, 210, 94-102.

NEISSER, U. Cognitive psychology. Nueva York. Appleton-Century-Crofts, 1967.

NORMAN, D. A. Memory while shadowing. Quarterly Journal of Experimental Psychology, 1969, 21, 85-93.

NORMAN, D. A., y RUMELHART, D. E. A system for perception and memory. En D. A. Norman (ed.). Models of human memory. Nueva York. Academic Press, 1970, págs. 21-64.

PALMER, S. E., y ORNSTEIN, P. A. Role of rehearsal strategy in serial probed recall. Journal of Experimental Psychology, 1971, 88, 60-66.

PETERSON, L. R. Short-term verbal memory and learning. Psychological Review, 1966, 73, 193-207.

PETERSON, L. R., y JOHNSON; S. T. Some effects of minimizing articulation on short-term retention. Journal of Verbal Learning and Verbal Behavior, 1971, 10, 346-354.

PETERSON, L. R., y KROENER, S. Dichotic stimulation and retention. Journol of Experimental Psychology, 1964, 68, 125-130.

PHILLIPS, W. A., y BADDELEY, A. D. Reaction time and short-term visual memory. Psychonomic Science, 1971, 22, 73-74.

POSNER, M. I. Short-term memory systems in human information processing. Acta Psychologica, 1967, 27, 267-284.

POSNER, M. I. Abstraction and the process of recognition. En G. H. Bower y J. T. Spence (eds.). The psychology of learning and motivation: Advances in research and theory, Vol. III. Nueva York. McGraw Hill, 1969, págs. 152-179.

POSTMAN, L. Short-term memory and incidental learning. En A. W. Melton (ed.). Categories of human learning. Nueva York. Academic Press, 1964, págs. 145-201.

ROSENBERG,.S., y SCHILLER, W. J. Semantic coding and incidental sentence recall. Journal of Experimental Psychology, 1971, 90, 345-346.

RUNDUS, D. Analysis of rehearsal processes in free recall. Journal of Experimental Psychology, $1971,89,63-77$

SAVIN, H. B.. Y BEVER, T. G. The nonperceptual reality of the phoneme. Journal of Verbal Learning and Verbal Behovior, 1970, 9, 295-302.

SCHULMAN, A. I. Recognition memory for targets from a scanned word list. British Journal of Psychoiogy, 1971, 62, 335-346. 
SELFRIDGE, O. G., y NEISSER, U. Pattern recognition by machine. Scientific American, 1960, 203, 60-68.

SHAFFER, W. O., y SHIFFRIN, R. M. Rehearsal and storage of visual information. Journal of Experimental Psychology, 1972, 92, 292-296.

SHALLICE, T., y WARRINGTON, E. K. Independent functioning of verbal memory stores. A neuropsychological study. Quarterly Journal of Experimental Psychology, 1970, 22, 261-273.

SHEPARD, R. N. Recognition memory for words, sentences, and pictures. Journal of Verbal Learning and Verbal Behavior, 1967, 6, 156-163.

SHIFFRIN, R. M., y ATKINSON, R. C. Storage and retrieval processes in long-term memory. Psychological Review, 1967, 76, 179-193.

SHULMAN, H. G. Encoding and retention of semantie and phonemic information in short-term memory. Journal of Verbal Learning and Verbal Behavior, 1970, 9, 499-508.

SHULMAN, H. G. Similarity effects in short-term memory. Psychological Bulletin, 1971, 75, 399-415.

SHULMAN, H. G. Semantic confusion errors in short-term memory. Journal of Verbol Learning and Verbal Behavior, 1972, 11, 221-227.

SILVERSTEIN, C., y GLANZER, M. Concurrent task in free recall: Differential effects of LTS and STS. Psychonomic Science, 1971, 22, 367-368.

SPERLING, G. Short-term memory, lont-term memory, and scanning in the processing of visual information. En A. Young y D. B. Lindsley (eds.). Early experience and visual information processing in perceptual and reading disorders. Washington. National Academy of Sciences, 1970, págs. 198-215.

STOFE, M., y EAGLE, M. N. The relationship among reported strategies, presentation rate, and verbal ability and their effects on free recall learning. Journal of Experimental Psychology, $1971,87,423-428$.

SUTHERLAND, N. S. Outlines of a theory of visual pattern recognition in animals and man. Proceedings of the Royal Society. Serie B, 1968, 171, 297-317.

TKEISMAN, A. Monitoring and storage of irrelevant messages in selective attention. Journal of Verbal Learning and Verbal Behavior, 1964, 3, 449-459.

TRESSELT, M. E., y MAYZNER, M. S. A study of incidental learning. Journal of Psychology, $1960,50,339-347$.

TULVING, E. Subjective organization and effects of repetition in multi-trial free-recall learning. Journal or Verbal Learning and Verbal Behavior, 1966, 5, 193-197.

TULVING, E., y MADIGAN, S. A. Memory and verbal learning. Annual Review of Psychology, $1970,21,437-484$.

TULVING, E., y PATTERSON, R. D. Functional units and retrieval processes in free recall. Journal of Experimental Psychology, 1968, 77, 239-248.

TULVING, E., y PEARLSTONE, Z. Availability versus accessibility of information in memory for words. Journal of Verbal Learning and Verbal Behavior. 1966, 5, 381-391.

TURVEY, M. T. Repetition and the preperceptual information store. Journal of Experimental Psychology, 1967, 74, 289-293.

WARRINGTON, E. K. Neurological disorders of memory. British Medical Bulletin, 1971, 27, 243-247.

WATKINS, M. J. The characteristics and functions of primary memory. Tesis doctoral inédita. Universidad de London, 1972.

WAUGH, N. C., y NORMAN, D. A. Primary memory. Psychological Review, 1965, 72, 89-104.

WICKER, F. W., y BERSTEIN, A. L. Association value and orienting task in incidental and intentional paired-associate learning. Journal of Experimental Psychology, 1969, 81, 308-311. 